\title{
QUERIDO CUADERNOS DE PEDAGOGÍA. UNA MIRADA DESDE LA AÑORANZA
}

\section{Dear Cuadernos de Pedagogía. A glance from the yearning}

\author{
Luis Torrego EgIDo \\ Universidad de Valladolid. Facultad de Educación. Segovia \\ Correo-.e: luis.torrego@uva.es
}

Recibido: 3 de noviembre de 2020

Envío a informantes: 9 de noviembre de 2020

Aceptación definitiva: 2I de diciembre de 2020

Resumen: Cuadernos de Pedagogía ha sido la revista profesional que mayor impacto e influencia ha tenido en la educación española en los últimos cuarenta años. La cabecera sigue en el mercado, pero sus impulsores y su espíritu ya no están en la revista. En este artículo se rememora a sus principales promotores y las características significativas de la revista, así como alguno de los contenidos y las iniciativas que la publicación puso en marcha. Se hace desde la mirada, necesariamente subjetiva, del autor, que ha sido acompañado por la revista y reconoce su influencia en la conformación de su identidad docente. Hoy, en tiempos de escuela de pandemia, añora una revista que tiene entre sus logros su contribución a la renovación educativa, a la definición de las señas de identidad de la escuela pública democrática, a la difusión de la memoria pedagógica, al acercamiento de la teoría y de la práctica y al debate pedagógico.

Palabras clave: Cuadernos de Pedagogía; prensa pedagógica; renovación pedagógica; escuela pública; formación del profesorado.

Aвstract: Cuadernos de Pedagogía has been the professional journal that has had the greatest impact and influence on Spanish education in the last forty years. The masthead is still on the market, but its makers and its spirit are no longer in the journal. This paper recalls its main promoters and the significant characteristics of the journal, as well as some of the content and initiatives that the publication set in motion. This is done from the necessarily subjective viewpoint of the author, who has been accompanied by the journal and recognises its influence in shaping his or her teaching identity. Today, 
in times of pandemic school, he longs for a journal that has among its achievements its contribution to educational renewal, to the definition of the hallmarks of the democratic state school, to the dissemination of pedagogical memory, to the approach of the theory and practice and pedagogical debate.

KeY words: Cuadernos de Pedagogía; pedagogical press; state school; pedagogical renewal; teacher training.

\section{Introducción}

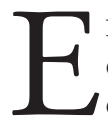
N LAS LÍNEAS QUE SIGUEN ofrezco una visión, subjetiva y necesariamente parcial, de la revista Cuadernos de Pedagogía y de su influencia en la conformación de mi conciencia pedagógica. Lo hago con una doble intención que ya declaro: por un lado, realizar un acto de clarificación de ese ideario que creo que puede tener un cierto sentido de representación de algún rasgo no solo individual, sino colectivo; por el otro, mostrar gratitud ante el compromiso que, como trataré de exponer, la revista tuvo con la renovación educativa. He dicho tuvo porque quiero aclarar que esta rememoración se refiere a la revista que inició su andadura en los estertores del franquismo y no a la que hoy lleva su mismo nombre, que no tiene detrás ni las mismas personas ni, claramente, el mismo espíritu. Si este trabajo no llegara a alcanzar esos propósitos, creo que servirá, al menos, aunque sea de manera desordenada, para evocar algunas de las notas más distintivas de esta joya de la prensa pedagógica que fue la revista.

Comencé mis estudios de Magisterio en la entonces denominada Escuela Universitaria de Formación del Profesorado de EGB «Virgen de la Fuencisla» de Segovia, un centro público que formaba parte de la Universidad Autónoma de Madrid. Era el año I977 y yo no era consciente del intenso ambiente de ebullición pedagógica que se estaba desarrollando en nuestro país. Sin embargo, fui descubriendo algunas cosas que me ocasionaron un deslumbramiento vocacional, un intenso deseo de conocer y paladear aquellas ideas nuevas que hablaban de una educación llena de belleza y de posibilidades humanizadoras, de ingredientes para la construcción de una sociedad más justa.

Recuerdo bien que, quizás empujado por una formación rutinaria, en clases masificadas, llegué a hacer novillos para leer a Paulo Freire y su Pedagogía del Oprimido. Ese estudiante aún casi adolescente que era yo entonces, en un viaje a la cercana $\mathrm{Ma}-$ drid, descubrió en una librería un ejemplar de una revista de educación, que hojeó y compró, llevado de la curiosidad. Asíllegué a Cuadernos de Pedagogía, que me ha acompañado desde entonces hasta hace tres años.

\section{El nacimiento de la revista}

Cuadernos de Pedagogía había aparecido casi tres años antes, en enero de 1975, con una «atractiva portada, 48 páginas y su precio era de 50 pesetas. De aquel número I se imprimieron 7.000 ejemplares, agotados en la actualidad» (Palacios, 1985, p. 9). El primer párrafo de su editorial, de carácter programático, deja claras las aspiraciones y los rasgos definitorios de la revista: 
Cuadernos de Pedagogía se dirige, ante todo, a hacer públicas las ambiciones de renovación escolar y, por tanto, social; a dar a conocer las reflexiones e inquietudes de todos los profesionales de la enseñanza, a reunir ese disperso pensar individual, o de núcleos más o menos amplios, para que se reencuentren en un espacio común y se transformen en un hacer colectivo.

El editorial prosigue enumerando los objetivos que se propone: «La reflexión, el intercambio, la polémica, la crítica y las alternativas para la construcción de una enseñanza popular y científica, para una defensa intelectual y material del profesional de la misma» e inmediatamente después refleja una autodefinición de la publicación: «... es, pues, sólo un proyecto que ahora se inicia y cuya viabilidad está un poco en manos de todos y cada uno de los enseñantes de los diversos lugares de España». En las frases del editorial ya se apuntan las finalidades y los principios de la revista, que seguirá con empeño y que continuarán caracterizándola durante más de cuatro décadas.

La revista nace unos meses antes de la muerte de Franco, en pleno período de implantación de la Ley General de Educación, que venía precedida de un documento de diagnóstico muy amplio y concreto, el Libro Blanco, realizado antes de promulgar la ley. La ley, pese a elaborarse dentro de un régimen dictatorial, es modernizadora y recoge influencias internacionales, de modo que «el estudio a fondo de su gestión permite descubrir pretensiones democráticas» (Serrano, 20I5, p. 259). La educación del franquismo, la que yo viví, era mucho más antigua que la que regulaba la nueva ley y queda retratada de forma meridiana en los versos de una canción del cantautor José Antonio Labordeta:

Dulcemente educados,
en tardes de pavor,
conteniendo la risa,
el grito y el amor,
sin comprender la fuerza
de un viento abrasador.
Fuimos creciendo en filas
de dos en dos,
cruzando las ciudades,
los barrios, la ilusión,
dejando todo atrás
sin comprensión. (Labordeta, 1976)

Es evidente que el panorama educativo resultaba entonces insatisfactorio para quienes lo conformaban. Por eso, la aparición de la revista se acompaña de un contexto en el que pueden resaltarse una extraordinaria conflictividad universitaria, que lleva incluso a la suspensión del curso en la Universidad de Valladolid, cerrada en febrero de 1975 , o los conflictos laborales en los institutos de Enseñanzas Medias, con el profesorado no numerario reclamando mejoras. El panorama se completa con el comienzo de la proliferación de escuelas de verano de los movimientos de renovación pedagógi$\mathrm{ca}$, en las que, entre otras cosas, se abordan temas generales de política educativa (y de política general, con un afán democratizador) y se buscan nuevos métodos de trabajo y nuevas prácticas organizativas. La insatisfacción sobre la formación recibida es, en 
general, grande y desde instancias oficiales -organismos ministeriales, centros educativos, revistas- no hay capacidad para colmar las ansias de transformación educativa.

Justo en ese momento surge Cuadernos de Pedagogía, una revista que, desde sus primeros números, tiene como señas de identidad la defensa de la escuela pública, una escuela pública igualitaria y de calidad, de una enseñanza científica y enraizada en el entorno, la articulación de un sistema educativo democrático e integrador y la dignificación del profesorado y de la formación de este. Aparece claramente explícita su vinculación con la renovación pedagógica (y, por tanto, social, como se dice en el primer editorial) y la pretensión de constituirse en una plataforma del y para el profesorado, una plataforma que no quiere ser académica, sino cercana a la vida del profesorado. Estas aspiraciones, como tendremos ocasión de mostrar, no son un mero enunciado, sino logros de la revista.

La revista tiene a Fabricio Caivano como redactor jefe y a Jaume Carbonell como secretario de redacción. Se trata de dos personas extraordinariamente comprometidas con la renovación pedagógica, que ejercerán la dirección de la revista durante muchos años. «Jaume -alto, pausado, calmoso- y Fabricio - pequeño, inquieto, nervioso, anticipándose a lo que se iba a decir-forman una pareja que se complementa perfectamente y que constituyen un equipo de trabajo de eficacia inigualable» (Delval, 1997). Esa eficacia se basa en que no se limitaron a buscar o seleccionar artículos, sino que, como señala el propio Delval, se los podía ver en cada acontecimiento educativo, en debates o jornadas, visitando centros educativos innovadores o modestas escuelas rurales para recoger voces y ofrecer una imagen viva de las esperanzas educativas.

\section{Una revista del y para el profesorado, comprometida con la renovación educa- tiva}

Cuadernos de Pedagogía se ha caracterizado por ser una empresa colectiva, pues han sido los maestros y maestras, los profesores y profesoras, los que se han hecho dueños de la publicación, con la autoría de los artículos, con su difusión, convirtiéndose, tal y como señaló Jesús Palacios (1985), en espejo, aglutinante y motor de los esfuerzos por transformar la educación en España. La revista logró su pretensión de convertirse en un cauce de información para el profesorado, pero también la de ser un espacio para el debate, para el contraste de opiniones y, sin abandonar ninguna de ellas, la de servir como plataforma para el intercambio y difusión de experiencias.

Cuadernos tiene el mérito de haber sabido recoger el saber práctico del profesorado, de los maestros y maestras, que han enviado a la revista reflexiones y, sobre todo, experiencias y propuestas para construir la escuela pública democrática, más allá de discursos teóricos (que también los ha habido en la revista) y formulaciones abstractas. Se visualiza, así, otro modelo de escuela, hecha posible desde experiencias reales. Jaume Carbonell relata una frase de un catedrático de universidad, de Pedagogía, que decía «esta revista no puede tener mucho nivel porque participan demasiados maestros, escriben demasiados maestros» (Carbonell, 2013). Coincido con él en señalar esta como una de las principales virtudes de la publicación. Hoy, cuando la servidumbre a los índices de impacto y al capitalismo académico se ha interiorizado y forma parte de la lógica cotidiana de quienes trabajamos en las universidades, volver la mirada a esos contenidos de Cuadernos sigue siendo un motivo para la esperanza de recobrar 
alguna vez el sentido común y la esperanza de un trabajo útil, digno y no alienado, con el norte puesto en la buena educación y no en los intereses del mercado ni en los supuestos beneficios de la competitividad.

Me gustaría resaltar, especialmente, ese carácter de punto de encuentro para dar cabida a temas e inquietudes transformadoras. Un punto de encuentro no neutral, sino muy comprometido con la renovación, invitando siempre a su puesta en práctica con ideas para el trabajo teórico y herramientas para el trabajo práctico. Delval (I997) señala este carácter colectivo: «La mayor parte de los lectores no saben quiénes han sido los siete propietarios que se han sucedido, ni posiblemente les ha interesado». Sin embargo, este asunto de la propiedad tendrá una importancia crucial en la trayectoria de la revista. Así, la palabra empresa que hemos utilizado al comienzo de este párrafo con la acepción de designio de llevar a cabo un proyecto pasará con el tiempo a trasladar su significado a la unidad de organización dedicada a actividades mercantiles con fines lucrativos, como veremos. Y ahí empezará a torcerse el rumbo de esta singladura pedagógica.

Al estudiante que era yo entonces, en una pequeña capital de una provincia aun más pequeña, alejado de las influencias directas de movimientos de renovación pedagógica o del contacto con figuras relevantes e innovadoras de la educación, la lectura de los sucesivos números de la publicación le supusieron un aldabonazo en sus esquemas pedagógicos. Surgió en mí el descubrimiento de imágenes e ideas educativas en las que, poco a poco, iba profundizando e iba formándome en mi identidad docente, percibiendo con claridad, con objetividad basada en argumentos, cómo debía ser esa educación que quería y conectándola con mi subjetividad, con mis ideales y con la esperanza. Y no solo eso: en la revista encontraba también realizaciones prácticas de esas ilusiones didácticas. Podría afirmar que esta revista fue un claro influyente de mi politización educativa, de mi preocupación y mi compromiso con la buena educación. No es extraño que, una vez suscrito, esperase con impaciencia la llegada de un nuevo número a mi buzón.

En medio de estas zozobras, en el momento en el que escribe el inspector de Educación Jesús Jiménez Sánchez, puede afirmarse, como él lo hace, que la publicación «ha sido, seguramente, la revista profesional que mayor impacto e influencia ha tenido en la educación española en los últimos cuarenta años» (Jiménez Sánchez, 20I5, p. I2). O, yendo más allá, coincidir con Vázquez Montalbán (1997) cuando afirma que Cuadernos de Pedagogía es algo más que una revista especializada, una de las herramientas de construcción crítica de la democracia cultural española a partir de 1975. En efecto, en opinión de quien escribe, la revista ha alimentado el esencial optimismo pedagógico, pues ha ayudado a extender en las personas que la hemos leído la idea de que la escuela puede contribuir decisivamente a la transformación social. Para esa transformación resulta esencial la renovación educativa y Cuadernos de Pedagogía fue una extraordinaria palanca de esa renovación. Trataré de justificar esta afirmación.

Sus impulsores escriben en 1979 un artículo sobre la renovación pedagógica con una perspectiva histórica y comparada, atribuyendo sus inicios a la Institución Libre de Enseñanza, definiendo la renovación pedagógica como «un conjunto organizado de actividades teóricas y prácticas dirigidas a combatir y a superar la teoría y la práctica pedagógica dominantes en un momento dado» (Caivano y Carbonell, 1979, p. 4).

A esa definición se puede añadir la que da Luis Miguel Lázaro (2003), para quien la renovación pedagógica resulta de la conjunción armónica y complementaria de la 
innovación educativa y la acción política orientada a la transformación progresiva y simultánea de la sociedad y de la educación. Añadamos las notas que, según Santiago Esteban (2016), en un estudio sobre los movimientos de renovación pedagógica, caracterizan esa renovación pedagógica: la crítica a la escuela tradicional, la defensa de una escuela democrática y cívica, el apoyo al laicismo y la coeducación; la configuración de un espacio para el desarrollo de un modelo de profesor educador, vocacional, humanista y científico; dar espacio a corrientes pedagógicas transformadoras y a autores relevantes en el campo internacional y el análisis riguroso de las políticas educativas oficialistas. Pues bien, no cabe duda de que Cuadernos de Pedagogía cumple los requisitos que establecen Esteban y Lázaro y los que enunciaron sus directores para ser considerado un elemento generador de esa renovación educativa de la que venimos hablando.

Pero, además, es preciso señalar otro rasgo que mantuvo en sus primeras décadas la revista: la defensa de los ideales renovadores no es gratuita ni ciega; se hace sin abandonar una postura crítica. Para ilustrar esto sirve su posición a favor de la escuela pública, mostrada en editoriales y en la selección de artículos y experiencias, sin ignorar que la escuela pública de la que se habla es un proyecto no realizado y envuelve una realidad con miserias, inclinada en ocasiones al modelo de escuela pública estatal, invadida por el poder político, y en ocasiones al modelo de escuela corporativa, secuestrada por los intereses particulares del profesorado. Esos modelos de escuelas han sido denunciados en la revista, que ha prestado sus páginas para abogar por la escuela pública comunitaria, que es abierta y se construye con la participación de la comunidad educativa, que es garante de los derechos del alumnado a una educación inclusiva y compensadora, y que está enraizada y vinculada a su entorno.

Por si alguien quiere detenerse en comprobar lo que se expone en el párrafo anterior, aquí va una pequeña selección personal, recomendada por quien escribe, de artículos que muestran, además, la permanencia de la reivindicación de esa añorada escuela pública: «¿Qué cuestan la enseñanza pública y privada?», José Luis Bernal Agudo (1985, n. ${ }^{\circ}$ I23); la entrevista titulada «Emilio Lledó; Baluarte de la enseñanza pública» (2000, n. ${ }^{\circ}$ 287); «Escuela pública: el libre pensamiento», Luis Gómez Llorente (200I, n. ${ }^{\circ}$ 30I); «Opinión. La educación pública como cultura», José Gimeno Sacristán (20I2, n. ${ }^{\circ}$ 426), o los más recientes «Escuela pública y derecho a la educación», de Julio Rogero Anaya (2014, n. $\left.{ }^{\circ} 443\right)$ o «Utopías pedagógicas: logros y frustraciones», de Jaume Martínez Bonafé (2014, n. $\left.{ }^{\circ} 45 \mathrm{I}\right)$.

\section{Las etapas de la revista}

Los primeros años son de precariedad material -la revista se había fundado con un capital de apenas 300.000 pesetas-, pero de libertad ideológica, «de la mano de pequeños editores como Frederic Pagés o la editorial Fontalba, en la que estuvieron siete años» (Pérez Oliva, 1997). En 1995 RBA Editores, un potente grupo editorial, compró a Fontalba todas sus publicaciones. Ahí comenzó una etapa triste para los impulsores de la revista que, según Milagros Pérez Oliva, reclamaron entonces su regularización laboral. RBA les reconoció, tras una larga negociación, diez años de antigüedad. En ese momento, 1997, «sin previo aviso y sin haberlo siquiera intuido, el editor les anunció que la revista y ellos mismos habían sido vendidos a Praxis» (Pérez Oliva, 1997). Praxis formaba parte del grupo holandés Wolters Kluver, una empresa especializada 
en libros jurídicos y guías legales que se ha expandido a temas fiscales, financieros, de recursos humanos y ha penetrado en los ámbitos educativos y sanitarios.

En febrero de 1997 el director de la publicación, Fabricio Caivano, es despedido, con una escueta carta, por motivos disciplinarios, tras 22 años de gestión. La gota que colma el vaso es una entrevista a la entonces ministra de Educación, Esperanza Aguirre, cuyo cuestionario, elaborado por Caivano, exige supervisar la editora. Vázquez Montalbán (1997) sintetiza atinadamente lo ocurrido, diciendo que los nuevos propietarios «van a pasar por encima del cadáver de Fabricio Caivano [...] arrollado por ejecutivos de acero inoxidable, insensibles a que este tipo de publicaciones son lo que son debido a una artesanía del espíritu difícil de introducir en las calculadoras».

Jaume Carbonell, por su parte, continuará en la revista hasta el año 2012, momento en el que se jubila, y se despide con un editorial titulado "Adiós y gracias». En una entrevista que se publica en la propia revista, en enero de 2013 , Jaume Carbonell afirma que Cuadernos de Pedagogía tenía una línea que era difícil de cambiar y que, aunque no fue presionado, llevado de su incomodidad por la dirección en solitario de la revista, volvió a recurrir al carácter colectivo que había marcado a Cuadernos: «Se me ocurrió la idea de gestar una red de corresponsales, buenos conocedores del mundo educativo con un perfil periodístico, para enriquecer los contenidos informativos» (Carbonell, 20I3, p. 5I).

Jaume Carbonell, en el año 200o, en un artículo de Martí Font en el diario El País, hace balance de la trayectoria de Cuadernos de Pedagogía hasta ese momento y distingue cuatro etapas diferentes:

a) La etapa utópica de los años setenta que dura hasta la llegada del PSOE al gobierno, en 1982, es la de proyectar sueños y buscar la utopía, con una gran efervescencia y un deseo de recuperar la memoria de la educación transformadora, sepultada por el franquismo. Son años, sin embargo, de autarquía pedagógica: no había conexión con lo que sucedía fuera del país.

b) La llegada del PSOE al poder supone que muchos profesores y maestros alcancen a dirigir la Administración educativa y a ella llegan las reivindicaciones históricas enunciadas en la etapa anterior. Se producen muchos cambios, pero también comienza la insatisfacción, pues algunos de los sueños son sepultados por una lógica gris.

c) La tercera etapa hay que situarla en torno al debate y aprobación de la Ley Orgánica de Ordenación General del Sistema Educativo (LOGSE) y su posterior aplicación. Es el momento de la verdad y Cuadernos de Pedagogía impulsa un debate a fondo sobre el tema, convirtiéndose realmente en una plataforma de debate.

d) La cuarta fase de Cuadernos de Pedagogía ha empezado en el año 200o. Se abre ahora el gran debate sobre las medidas de política educativa y social que deben tomarse para corregir aquellas cosas que no funcionan de la reforma.

En ese año 2000, la difusión media de la publicación, según datos de la OJD, es de I8.455 ejemplares, de los que i5.000 corresponden a suscripciones (Martí Font, 2000). En 2005 esa cifra de suscriptores ha bajado a I2.000.

Podría añadirse una quinta etapa, que vendría determinada por la Ley Orgánica de Mejora de la Educación, la LOMCE, una ley que se reflejó en las páginas de la revista, pero que tuvo un tratamiento diferente al dado a la LOGSE, pues no se trató tanto de 
debatir como de proponer críticas y alternativas, y por la crisis económica y su presencia en la escuela, a la vez que aparecía la innovación como gran tema de desarrollo, con un intento claro por no caer en las modas de la innovación vista como escaparate. Esta última etapa se extiende hasta que la Fundación Trilema se hace con la revista, momento en el que se amputa el espíritu de la publicación.

\section{Algunos hitos de Cuadernos de Pedagogía en mi memoria}

Hay algunos hitos en la revista que he seguido con especial atención y que considero necesario destacar, pues, además de poner de manifiesto mis intereses, creo que muestran también la importancia que ha tenido la revista.

Comencé a trabajar como maestro en pequeñas localidades de la provincia de Segovia -Sebúlcor, Fuenterrebollo, Sacramenia- y mi primera realidad profesional fue esa escuela que tiene tan poca presencia en los textos oficiales: la escuela rural. Sin embargo, en Cuadernos de Pedagogía ya se habían publicado entonces (finales de 1982) II6 artículos sobre la escuela rural, de los 263 que Lidia Sala y Mónica Simón (20I2) contabilizan en su estudio sobre los veinte primeros años de la revista. Como ellas dicen, la mayoría de los artículos provienen de las comunidades más rurales: Andalucía, Aragón, Castilla y León, Cataluña y Galicia. Esta es otra de las características de la revista: su vinculación a los territorios concretos y a las realidades educativas de cada uno de ellos. Puede decirse que en esto también encontré compañía en mi quehacer profesional.

En el año 1995 aparece un CD-ROM, 20 años contigo, que contiene los artículos publicados en los veinte años de la revista. Cinco años después, ese CD-ROM, que ha venido actualizándose año a año y pasa a ser 25 años contigo, contiene, nuevamente, toda la historia de la revista y acompaña a otro CD-ROM, titulado Comentarios y libros, que recoge todas las críticas publicadas sobre materiales educativos. Con motivo de esos 25 años, se inicia la publicación de un coleccionable que, con el título de $P e-$ dagogías del siglo $X X$, recoge la obra de los autores más influyentes en el pensamiento y la práctica educativa, desde Montessori a Piaget. En 2005, para conmemorar el $30{ }^{\circ}$ aniversario, la publicación vuelve a editar otros complementos interesantes: un coleccionable mensual titulado La escuela desorientada, que se compone de in litografías del artista gráfico Joma, y el libro Retratos de maestras. De la Segunda República hasta nuestros días, en el que se narra la experiencia de 30 profesoras de tres generaciones distintas, reflejando las transformaciones educativas desde una perspectiva sensible e innovadora.

La revista también ha acogido aportaciones polémicas. Ninguna más intensa que la generada por la publicación del artículo «¿Es pública la escuela pública?», del catedrático Mariano Fernández Enguita, en el ejemplar correspondiente a octubre de 1999, en el que responsabiliza al profesorado del deterioro del sistema educativo público. Se produce una multitud de reacciones a favor y en contra, algunas de ellas publicadas en sucesivos números de la revista. La disputa se desplaza después a un foro de debate en Internet, creado por el propio autor en un espacio de la Universidad de Salamanca, donde trabajaba entonces. La recopilación de esa intensa polémica se recogió en un libro, que tiene el mismo título del artículo y fue publicado por la editorial Praxis en 2002 (Fernández Enguita, 2002). 
En la revista he leído entrevistas a personalidades no solo del ámbito educativo. Fueron muy difundidas sus entrevistas a Felipe González o la ya citada de Esperanza Aguirre, así como las mantenidas con Jean Piaget, Manuel Vázquez Montalbán o con Maruja Torres. Recuerdo, especialmente, algunas otras como las de José Antonio Labordeta, Fernando Savater e Iñaki Gabilondo. En mi memoria quedan también las mantenidas con José Gimeno Sacristán y con Fernando Cardenal, en 1985; con John Elliott, en 1989, y la realizada por Juana M. ${ }^{a}$ Sancho y Francisco Hernández a Andy Hargreaves, en I995, o la de Emilio Lledó, en el año 2000.

Buceando en la nostalgia vienen a mí ahora secciones que cada vez me parecen más enriquecedoras, como los «Cuadernos de Paz», en los que aprendí el enfoque socioafectivo de la mano de Rafael Grasa o de la de Xesús Rodríguez Jarés, o esa fuente de recursos de la educación científica que se desplegaban en los «Cuadernos de Ciencia». Recuerdo mi mirada detenida en la sección dedicada a la experiencia del mes, donde tuve noticia de realizaciones educativas extraordinariamente valiosas, que mostraban que otra educación era -y es- posible, o el dosier, del que, para no agotar al lector o lectora, mencionaré únicamente uno, dedicado a la pedagogía operatoria, que para mí fue todo un descubrimiento. No puedo dejar de traer aquí la sección «Debate/La Reforma», que supuso un auténtico ejercicio democrático de discusión de los presupuestos ideológicos, de las bases pedagógicas y del contexto de la reforma educativa que pretendía realizar la LOGSE, de I990.

He seguido con particular interés las noticias de actividades y experiencias relacionadas con el Movimiento Cooperativo de Escuela Popular (MCEP) y la difusión de las realizaciones de la pedagogía Freinet en España, así como la atención dedicada a los encuentros y a los congresos de la Confederación de Movimientos de Renovación Pedagógica y a la difusión de sus propuestas. También me ha llegado el reflejo de experiencias de otra escuela, como, por citar solo algunos ejemplos, las comunidades de aprendizaje, las experiencias de pedagogía libertaria o la exposición de la organización de las escuelas Amara Berri.

En la mejor tradición de la prensa pedagógica -recuérdense los libros editados en el primer tercio del siglo xx por la Revista de Pedagogía, de Luzuriaga; por la Escuela Moderna, de Ferrer Guardia, o por Escuelas de España, de Bayón, Cobos y Hernanzla revista Cuadernos de Pedagogía de Caivano y Carbonell ejerció durante décadas un destacado papel en cuanto al impulso de la edición de libros, bien con un sello propio, o bien a través de colecciones específicas de editoriales próximas en cuanto a la filosofía de trabajo: es el caso, entre otras, de Laia, Paidós, Icaria, Reforma de la Escuela o Nuestra Cultura. En la colección más propia, denominada Libros Cuadernos de Pedagogía, podemos encontrar, por citar solo algunos que llegaron a mis manos, desde una obra de Célestin Freinet -El equilibrio mental del niño- hasta obras de Mario Lodi-Crónica pedagógica e Insieme-, pasando por otras de temática específica, como La escuela unitaria, de Jesús Jiménez, o de política educativa, como La escuela pública comunitaria, de Luis Gómez Llorente y Victorino Mayoral.

No me gustaría que se me quedase en el tintero que Cuadernos también me ha acompañado en mi etapa de profesor universitario. He asaltado la hemeroteca en muchas ocasiones para servirme de sus ideas y llevarlas a mis clases. La más utilizada, sin duda, ha sido el «Tema del Mes» publicado en el n. ${ }^{\circ}$ 3II, en 2002, coordinado por José Gimeno Sacristán, titulado «Iguales y diferentes» en el que se realiza un análisis de los conceptos igualdad y diversidad, para saber en qué contextos se utilizan y para qué 
fines, de manera que se esclarecen sus significados y se reflexiona sobre su utilización para caminar hacia una sociedad más justa y su relevancia en una escuela más innovadora, democrática y favorecedora del desarrollo de la autonomía del alumnado. En esas páginas me ha apoyado -y me sigo apoyando- para tratar de transmitir la verdad profunda de la normalidad de la diversidad, de que diversidad, diferencia y desigualdad no son sinónimos y de que la igualdad exige ir contra corriente.

\section{Epílogo}

En el año 20r8, por medio de un correo electrónico de Rocío Anguita, compañera de departamento, y a través de Jurjo Torres, me llegó la noticia de que la Fundación Trilema había comprado Cuadernos de Pedagogía. En la web de la Fundación se anuncia entonces, como si fuera el primer editorial programático, que su propósito principal será «crear opinión rigurosa sobre los aspectos que afectan al sistema educativo y ofrecer un sitio a los profesores para que encuentren los recursos necesarios para el desarrollo de sus competencias». Después aparecen los nombres del consejo de redacción, con «reconocidos profesionales [...]: Carlos Magro, vicepresidente de la Asociación Educación Abierta; José Antonio Marina, filósofo, escritor y pedagogo; Roser Batlle, pedagoga y presidenta de la Red Española de Aprendizaje-Servicio; Fernando Trujillo, profesor de la Universidad de Granada; Mariano Fernández Enguita, sociólogo y profesor de la Universidad Complutense; Alfonso González Hermoso, presidente de la Asociación Educación Abierta; y Dolors Reig, profesora y psicóloga social, entre otros». Al frente de la revista está la teóloga Carmen Pellicer.

Para advertir la magnitud del cambio, merece la pena detenerse en las áreas de actuación de la Fundación Trilema, según aparecen en su página web, para compararlas con las señas de identidad del proyecto de Cuadernos de Pedagogía en sus primeras décadas: coaching y evaluación del desempeño docente, consultoría y asesoramiento en gestión del cambio educativo según el Modelo Rubik, tutorización de proyectos emprendedores en las escuelas, creación y titularidad privada de centros educativos, elaboración de recursos didácticos, formación e investigación en pastoral y en el desarrollo de la interioridad (desde 2014 codirigen el Máster de Pastoral Educativa, en colaboración con Escuelas Católicas y La Salle de Aravaca, en Madrid).

Nada queda de aquel Cuadernos de Pedagogía, con su fuerza reveladora de la belleza de la educación como posibilidad de transformación, de avance hacia una sociedad más justa. Bueno, queda, no sé si como anécdota o como síntoma, la permanencia del provocador (Carbonell, 2002) Mariano Fernández Enguita, hoy alto cargo en el organigrama de la Administración, en su consejo de redacción. En el momento en que escribo estas líneas acaba de publicarse el número 513 y el editorial y el tema del mes se dedican al desarrollo de la competencia emprendedora (tema del mes: aprender a emprender). Es otro síntoma.

Qué necesario sería hoy, en estos tiempos de pandemia, disponer del espacio que fue Cuadernos, en una escuela cada día más cerrada y a la vez más expuesta a la penetración de intereses económicos, con nubarrones que amenazan la equidad y la inclusión, siempre débiles, por otra parte. Es cierto que por experiencia propia he recurrido en ocasiones a otras revistas pedagógicas -recuerdo ahora a Kikiriki. Cooperación Educativa- y que ahí están aún, aguantando el tipo, Aula de Innovación Educativa y 
las revistas de su grupo editorial o El Diario de la Educación, este último en versión online. Es crucial apoyar a estos medios.

Quizás sea verdad eso de que Cuadernos, nuestro Cuadernos, fue una revista generacional, como lo fueron las escuelas de verano, los movimientos de renovación pedagógica o la canción de autor, pero si así fuera no podemos permitirnos derrochar este caudal humanizador, tendremos que recordar y hacer nuestros el coraje y el empeño de Daniel Lefebvre, el excepcional director protagonista de Ça commence aujourd'bui, la premiada película de Bertrand Tavernier, con una conmovedora visión de las posibilidades de la educación, reflejado en su diario:

Hay cosas que nunca desaparecen, están en la carne, hablan, están en la tierra. Están en la tierra montones de piedras apiladas una a una, con las manos del padre, del abuelo. Toda su paciencia acumulada resistió a la lluvia, al horizonte, haciendo montoncitos ante la noche para retener la luz de la luna, para estar erguidos, para inventarse montañas y jugar con el trineo y creer que tocamos las estrellas. Se lo contamos a nuestros hijos, les diremos que fue duro pero nuestros padres fueron unos señores y que heredamos eso de ellos, montones de piedras y el coraje para levantarlas...

\section{Bibliografía}

Caivano, F. y Carbonell, J. (1979). La Renovación Pedagógica ayer y hoy. Cuadernos de Pedagogía, 59, s. p.

CARbonell, F. (2002). Palabras previas. En ¿Es pública la escuela pública? (pp. 13-28). Barcelona: Praxis.

Carbonell, J. (20I3). Aprendo muchísimo con las visitas a las escuelas/Entrevistado por Rafael Miralles. Cuadernos de Pedagogía, 430, 46-53.

Delval, J. (1997, 25 de febrero). Pedagogía sin 'Cuadernos'. El País.

Esteban, S. (2016). La renovación pedagógica en España: un movimiento social más allá del didactismo. Tendencias Pedagógicas, 27, 259-284.

Jiménez Sánchez, J. (2015). Cuadernos de Pedagogía y la escuela aragonesa. Forum Aragón, I4, I2-I4.

LabordetA, J. A. (1976). Rosa, rosae. En Cantes de la tierra adentro [LP]. Movieplay.

LÁzAro, L. M. (2003). Política y educación: la renovación pedagógica en España, 1970-1983 En E. Candeias (coord.), Actas do $V^{o}$ Encontro Ibérico de História da Educação. Renovação Pedagógica. Coimbra/Castelo Branco.

Martí Font, J. M. (2000, 2I de febrero). 25 años iluminando a enseñantes. El País.

Palacios, J. (1985). Introducción. En cuAdernos de Pedagogía (ed.). La educación en España según Cuadernos de Pedagogía (pp. 9-13). Barcelona: Laia.

Pérez Oliva, M. (1997, in de marzo). Polémica nueva etapa de 'Cuadernos de Pedagogía'. El País.

SALA, L. y Simón, M. (20I2). Escola rural: renovació, democratització i igualtat d'oportunitats. L'anàlisi de les transformacions de l'escola rural des de la revista «Cuadernos de Pedagogía» (1975-1995). En S. Marquès (pres.), XX Jornades d'Història de l'Educació. Cohesió social i educació (pp. I4I-162). Girona: Universitat de Girona.

Serrano, F. (20I5). La tramitación de la Ley General de Educación y Financiamiento del Sistema Educativo de 1970. Análisis desde una perspectiva politico-educativa. [Tesis doctoral]. Valencia: CEU.

Vázquez Montalbán, M. (1997, io de marzo). Pedagógico. El País. 
